\title{
Nanoconstructions on the base of double- stranded DNA molecules and their optical properties
}

\author{
S.G. Skuridin ${ }^{1}$, Yu.M. Yevdokimov ${ }^{1}$, D.P. Chulkov², V.M. Gusev², O.N. Kompanets ${ }^{2, *}$, and \\ F.V. Vereschagin ${ }^{2}$ \\ ${ }^{1}$ Engelgardt Institute of Molecular Biology RAS, 119991 Moscow, Russia \\ ${ }^{2}$ Institute for Spectroscopy RAS, 108840 Troitsk, Moscow, Russia
}

\begin{abstract}
Experimental results have been presented on studying optical properties of nanoconstructions formed of orientationally ordered neighbouring double-stranded DNA molecules in the structure of their liquid-crystalline phases and dispersion particles of these phases including ones cured with intercalators.
\end{abstract}

The aim of this work was to develop a method to determine the optical properties of orientationally ordered structures in the form of particles (nanostructures) of the cholesteric liquid-crystalline dispersion (CLCD) of double-stranded (ds) DNA in PEG-containing water-salt solutions [1] - this method proved to be the method of circular dichroism (CD).

The results of calculation of the optical activity of the ds DNA CLCD particles, carried out in [2], based on the theory of electromagnetic waves absorption with large molecular systems in the model, when the particle is regarded as a set of cells containing a large amount of absorbing radiation chromophores (nitrogen bases of DNA) and representing a single complex with a specific, changing the spiral direction of the polarizability, allowed to determine the value of the "structural" circular dichroism of DNA CLCD particles and its dependence on various physical parameters (a step of helical twist of "quasinematic" layers of neighboring DNA molecules, the particle size, and other parameters), and most importantly - allowed to come to the conclusion, according to which the formation of a spatially ordered CLCD particles structure must inevitably be accompanied by the appearance of an intense band in the CD spectrum. The amplitude of this band depends on the DNA concentration used to prepare the dispersion (in the range of 5 to $30 \mu \mathrm{g} / \mathrm{ml}$ ), the size of the particles themselves, the twist angle of layers, the distance between them, and the negative sign of this intensive band directly says that neighboring layers of right-spiral DNA molecules have a left spatial twist.

Applied in [2] approach has also been used to study the features of the CD spectra of DNA CLCD treated with some colored biologically active compounds (BAC), which can intercalated between pairs of DNA nitrogen bases and turn out to be included in the threedimensional structure of DNA CLCD particles.

\footnotetext{
* Corresponding author: onkomp@isan.troitsk.ru
} 
Design data were compared with the experimental results obtained by studying the influence of various factors on the DNA CLCD particles including BAC interacting with ds DNA. The optical properties in the UV region were examined for the samples of DNA CLCD and the same DNA dispersion treated with daunomycin (DAU) - "coloured" anthracycline antibiotic used in the treatment of many malignancies. Registration of the CD optical signal was performed with the CDS-2MUV dichrometer (the ISAN development) adapted for use with DNA nanostructures.

The experimental CD spectra showed that the formation of the DNA dispersion is accompanied by appearance of abnormal negative band in the CD spectrum (with a minimum at $\lambda \sim 270 \mathrm{~nm}$ ), located in the region of the absorption of nitrogenous bases, with the concurrence of its form to theoretically calculated one that leads to the unambiguous conclusion about the liquid-crystalline cholesteric packaging of DNA molecules in the dispersion particles - a particular property of molecules having geometrical and optical anisotropy.

The CD spectrum of DNA CLCD "coloured" by DAU comprises two abnormal bands having negative signs: one - in the absorption band of DNA nitrogen bases (at $\lambda \sim 270 \mathrm{~nm}$ ), and the other - in the absorption band of DAU chromophores (at $\lambda \sim 500 \mathrm{~nm}$ ). Coincidence of abnormal bands signs arranged in different regions of the spectrum suggests the DAU molecules intercalated between DNA base pairs with 90 degree inclination angle with respect to the long axis of the DNA molecule. If the amplitude of the abnormal band in the absorption region of the DNA nitrogen bases remains practically unchanged, the amplitude of the abnormal band in the absorption region of DAU molecules increases with the degree of their DNA binding. In fact, this relationship is a calibration curve (Fig. 1), which can be used to determine the DAU concentration in the DAU-containing sample solution, and the DNA CLCD itself is a biosensing unit that sensitively reacts to the BAC content in the solution.

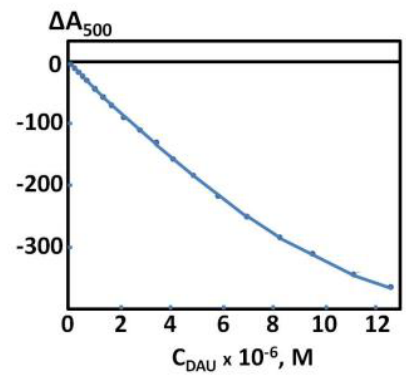

Fig. 1. The dependence of the amplitude of the $500 \mathrm{~nm}$ abnormal band in the $\mathrm{CD}$ spectrum of the CLCD particles of the complex (DNA-DAU) on the concentration of added DAU. C C DNA $=10 \mu \mathrm{g} / \mathrm{ml}$; $\mathrm{C}_{\mathrm{PEG}}=170 \mu \mathrm{g} / \mathrm{ml} ; \Delta \mathrm{A}_{500}=\left(\mathrm{A}_{\mathrm{L}}-\mathrm{A}_{\mathrm{R}}\right) \times 10^{-6}$ o.u.; $\mathrm{L}=1 \mathrm{~cm}$.

The support from the Program of RAS Presidium "Fundamental and applied problems of photonics and optical physics of new materials" is acknowledged.

\section{References}

1. Yu.M. Yevdokimov, V.I. Salyanov, S.G. Skuridin, Nanostructures and nanoconstructions based on DNA (CRC Press, Boca Raton, 2012)

2. Yu.M. Evdokimov, S.V. Semenov, S.G. Skuridin, V.I. Salyanov, P.N. Solev, V.T. Valuev - Elliston, F.V. Vereshchagin, D.P. Chulkov, O.N. Kompanets, Russian: Sensor Systems 30, 249 (2016) 